\title{
La acelerada búsqueda de candidatos terapéuticos contra SARS-CoV-2, métodos in silico: Revisión
}

\author{
The accelerated search for drug candidates against SARS-CoV-2, \\ in silico methods: Review \\ Oscar M. Cobar ${ }^{1,3,4 *}$ Rodrigo J. Vargas ${ }^{2,3,4}$ \\ ${ }^{1}$ Universidad del Istmo, ${ }^{2}$ Universidad Galileo, ${ }^{3}$ Red Latinoamericana de Implementación y Validación de Pautas \\ de Farmacogenómica Clínica (RELIVAF-CYTED), ${ }^{4}$ Unidad de Química Teórica y Computacional, \\ Facultad de Ciencias Químicas y Farmacia, Universidad de San Carlos, Guatemala
}

*Autor al que se dirige la correspondencia: ocobar@gmail.com

Recibido: 18 de agosto 2020 / Revisión: 03 de octubre 2020 / Aceptado: 12 de octubre 2020

Resumen

$\mathrm{E}$

1 reposicionamiento de fármacos como la derivatización química, que se han aplicado en los estudios de

descubrimiento y diseño de fármacos contra el SARS-CoV-2, dependen del ciclo de vida del virus, las dianas moleculares identificadas y un diseño basado en su estructura e interacciones moleculares. Se realizó una revisión extensa en las bases de datos públicas e institucionales RSCB-Protein Data Bank, ZINC, NCBI (PubMed, PMC), PubChem, Science Direct e instituciones como CDC, NIH y revistas científicas especializadas sobre los avances en la búsqueda de nuevas moléculas contra el nuevo coronavirus basadas en estudios in silico, detectándose más de 40,000 publicaciones sobre SARS-CoV-2 y cerca de 200 relacionadas a dichos estudios, las consideradas más relevantes fueron analizadas e incluidas en este artículo. Su análisis evidencia el avance acelerado de las herramientas computacionales y fortaleza del diseño de fármacos asistido por computadora (in silico approach) para la generación de nuevas moléculas con posibilidad de ser activas contra COVID-19 y presenta las principales dianas moleculares sobre la que actúan estos agentes con potencial antiviral.

Palabras claves: Estudios in silico, SARS-CoV-2, Mpro, Productos Naturales

\begin{abstract}
$\mathrm{T}$ he search of new applications for approved drugs by the regulatory authorities around the world, as well as their chemical derivatization in the search for new and effective drugs against SARS-CoV-2 depends of the viral life cycle, action of the drug and a receptor-based design. We performed a deep bibliographic research in peer reviewed scientific journals, data bases RSCB-Protein Data Bank, ZINC, NCBI (PubMed, PMC), PubChem, Science Direct and institutions (CDC, NIH) in the search of new molecules tested in silico against the novel coronavirus. As a result, we found more than 40,000 research papers related to SARS-CoV-2 and nearby 200 look on in silico studies, taking into consideration for this work all the more relevant for us, evidenced the accelerated advance and strength of the drug design assisted by computer (in silico approach) to develop new molecules that can be effective against COVID-19 and, at the same time, it exposes the main molecular targets.
\end{abstract}

Keywords: in silico approach, SARS-CoV-2, Mpro, Natural Products 


\section{Introducción}

La pandemia generada por COVID-19 sigue afectando al mundo mientras un tratamiento terapéutico efectivo o vacuna logre reducir su impacto. El desarrollo de fármacos es un proceso largo y complejo, que lleva muchos años de experimentación y distintas fases antes de ser sujeto a posibles ensayos clínicos. En la última década, gracias al avance de las tecnologías que impulsan la Química Computacional y Bioinformática, el desarrollo moderno de fármacos y su diseño asistido por computadora (diseño in silico) se ha convertido en una herramienta para alcanzar etapas clínicas en menos tiempo (Bruno et al., 2019).

La mejor oportunidad de éxito para un fármaco en ensayos clínicos dependerá de fases preclínicas robustas, incluido el diseño in silico. Hasta el momento, millones de moléculas han sido analizadas por computadora y tres dianas farmacológicas han sido exploradas contra el SARS-CoV-2. Moléculas de fármacos aprobados contra enfermedades virales como Ébola, VIH, MERS y SARS-CoV, antimicrobianos, antimaláricos y antiparasitarios e incluso productos naturales han sido estudiadas, promoviendo la síntesis de las mas promisorias, alcanzando varias de ellas resultados preliminares prometedores en estudios clínicos (Farag et al., 2020). La Main Protease (M ${ }^{\mathrm{PRO}}$ ), la ARN-dependiente ARN polimerasa (RdRp) y la proteína S del SARS-CoV-2 son hasta el momento, los objetivos terapéuticos más utilizados para orientar estos esfuerzos (Cava et al., 2020, Chamdel et al., 2020; Li $\&$ De Clerck, 2020; Oliveira et al., 2020; Wrapp et al., 2020). Los datos obtenidos de estudios in silico aportan evidencia preliminar a favor de favipiravir como el más prometedor (Sanders et al., 2020), sin descartar moléculas de origen natural que, de llegar a ensayos clínicos, sin duda, a nuestro criterio, serán validadas para el tratamiento de esta y otras enfermedades que azotan a la humanidad.

En este trabajo se realizó una extensa revisión de la literatura científica relacionada con la estructura del SARS-CoV-2, su mecanismo de ingreso a la célula humana, las estrategias terapéuticas actuales y estudios in silico de moléculas presentes en productos naturales y medicamentos aprobados para su utilización por la Administración de Drogas y Alimentos (FDA por sus siglas en inglés) de Estados Unidos y otras agencias reguladoras contra diferentes enfermedades, para estudiarlos contra el virus en la iniciativa de la
Organización Mundial de la Salud "Reutilización de Medicamentos" (Repurposing Drugs).

\section{Contenido}

\section{Diseño de fármacos}

El objetivo en el diseño de fármacos es encontrar moléculas que puedan convertirse en medicamentos contra las enfermedades que afectan a la humanidad. Para ello y alcanzar el éxito en los ensayos clínicos necesarios para comercializarlos, las compañías farmacéuticas, desde hace más de 15 años, han desarrollado diferentes estrategias para el descubrimiento de entidades farmacológicamente activas, siendo la identificación de objetivos terapéuticos, mejora de sus procesos de diseño y validación las mas importantes (Weinmann \& Metternich, 2005). En la actualidad, los enfoques en el diseño de fármacos se apoyan en la farmacogenética y epigenética, generación de nuevas herramientas computacionales, mejor conocimiento de los objetivos moleculares del potencial fármaco y el trabajo multidisciplinario, fundamentales en la optimización de los procesos de ingeniería de medicamentos y mayor probabilidad de éxito en los ensayos clínicos correspondientes (Ganesan et al., 2019).

En los últimos 30 años, el diseño de nuevos fármacos ha sido fortalecido por disciplinas como biología molecular, genética y farmacología entre otras, centrándose los avances en el entendimiento de mecanismos de acción y modulación de funciones del objetivo terapéutico (Monaghan \& Barrett, 2006). El tiempo promedio para la aprobación de un nuevo fármaco es de 7.2 años dependiendo del área terapéutica; desde 5.2 años para antivirales hasta 7.9 en antineoplásicos. El tomar en cuenta los estudios in silico, in vitro e in vivo previos, lo eleva hasta los 9 años. Sin embargo, se compensa con la cada vez mayor tasa de éxito $(16 \%)$ y la disminución significativa del costo estimado en 1.24 mil millones de dólares americanos. (Kaitin, 2010).

La prometedora y cada vez más importante etapa preclínica in silico es clave para el descubrimiento temprano de fármacos, al hacer mucho más eficiente la obtención de su estructura, su síntesis química y principalmente, el estudio de su interacción con el objetivo terapéutico, permitiendo la optimización de moléculas obtenidas de bancos de datos o bien, su diseño basado en homología estructural con medicamentos ya existentes (Bruno et al., 2019). 


\section{Ciclo de vida del SARS-CoV y estrategias terapéuticas}

Desafortunadamente, a la fecha, ningún medicamento o vacuna ha sido aprobado para el tratamiento de la enfermedad provocada por el SARS-CoV-2. Varias opciones se han evaluado para prevenir, controlar y curar el COVID-19, incluyendo terapia con plasma de convalecientes, vacunas, anticuerpos monoclonales, terapias basadas en oligonucleótidos, péptidos, interferón y medicamentos (Li \& De Clercq, 2020).

El ingreso del virus a la célula humana depende de su enlace al receptor celular, ACE2 y su fusión directa con la membrana celular (Boopathi et al., 2020; Hoffmann et al., 2020; Lukassen et al., 2020).

Para ello, su proteína S es escindida en dos subunidades S1 y S2 por la enzima TMPRSS2. S1 se enlaza con ACE2 como en el SARS-CoV (Li et al., 2005) y S2 se funde con la membrana celular.

A su ingreso, el virus libera su ARN en el citoplasma, es traducido en el ribosoma para generar las poliproteínas que luego participan en el proceso de transcripción y traducción del virus para generar sus proteínas estructurales y funcionales. Dichas proteínas se ensamblan dentro del aparato de Golgi vía su previa traducción en el retículo endoplásmico (Sanders et al., 2020).

Los principales objetivos terapéuticos son tres; el complejo ACE2-TMPRSS2, la 3-chymotrypsinlike-protease (3CL o $\mathrm{M}^{\mathrm{PRO}}$ ) y la $\mathrm{RdRp}$.

Al complejo ACE2-TMPRSS2 lo inhiben medicamentos como umifenovir (Arbidol $囚$ ) y los mesilatos de camostat y nafamostat, este último ha mostrado promesa en ensayos clínicos Fase III en curso.

Favipiravir (comercializado por empresas japonesas, chinas, rusas e indias) y remdesivir por Gilead Sciences de Estados Unidos inhiben a RdRp, siendo a la fecha los más promisorios, de acuerdo con resultados preliminares de ensayos clínicos Fase III (Sisay, 2020).

$\mathrm{M}^{\mathrm{PRO}}$ o $3 \mathrm{CL}^{\mathrm{PRO}}$ es inhibida por medicamentos como lopinavir, ritonavir y darunavir, siendo los dos primeros en combinación los más estudiados es ensayos clínicos con resultados poco prometedores ( $\mathrm{Li}$ et al., 2020).

Actualmente, se estudian combinaciones de fármacos con distintos mecanismos de acción antiviral para buscar su efectividad. Mesilato de nafamostat/ favipifravir, ribavirina, lopinavir-ritonavir e interferón beta $1 b$, remdesivir/baricitinib, remdesivir-dexametasona, favipiravir-dexametasona, entre otros (Li \& De Clercq, 2020).

\section{Genoma del SARS-CoV-2 y estructura de proteínas-objetivo}

El genoma del SARS-CoV-2 consta de 29,811 nucleótidos, que se estima expresan entre 25 y 29 proteínas (Kim et al., 2020), (GenBank: NC 045512.2) entre ellas sus proteínas estructurales Nucleocápsida (N), Membrana (M), Envoltura (E) y Spike (S).

La cápside es la capa de protección del virus que resguarda su material genético. En su interior se encuentra la proteína $\mathrm{N}$ enlazada con la cadena de ARN del virus, habilitándolo para ingresar a la célula humana y realizar los procesos de transcripción y replicación viral.

$\mathrm{Su}$ extremo N-terminal está unido al ARN genómico y subgenómico de los viriones MHV e IBV, lo que permite procesar su replicación y transcripción (Maranon et al., 2020).

La proteína $\mathrm{M}$ juega un papel importante en la fusión de la subunidad S2 de la proteína S con la membrana celular, adicionalmente a su función estructural dentro del virus de dar forma a su envoltura (Huang et al., 2020).

La proteína E es una glicoproteína, la más pequeña del virus, responsable de las propiedades conformacionales y de las interacciones proteína-proteína, importantes para el ingreso del virus a la célula.

Las proteínas E y M del SARS-CoV-2, comparten entre el 76 y $97 \%$ de identidad aminoacídica con SARS-CoV y la proteína expresada por el gen del virus del pangolín y la obtenida del murciélago chino (RaTG13) respectivamente (Bianchi et al., 2020).

\section{Estructura de la proteína $S$}

El SARS-CoV-2 utiliza la proteína altamente glicosilada $\mathrm{S}$ para ingresar a la célula. Es una proteína trimérica Fusión-Clase I, típica de los coronavirus, que existe en una conformación de prefusión metastable, que bajo rearreglos estructurales, es la responsable de la fusión del virus con le membrana de la célula humana (Bosch et al., 2003).

$\mathrm{Su}$ ingreso depende de su enlace al receptor celular ACE2 y su fusión directa con la membrana celular (Boopathi et al., 2020; Hoffmann et al., 2020; Lukassen et al., 2020).

TMPRSS2 como en el SARS-CoV, es la responsable de la escisión de la proteína S en S1 y S2 (Glowacka et al., 2011; Hoffmann et al., 2018; Matsuyama et al., 2010; Rensi et al., 2020; Shulla et al., 2011). S1 se 
enlaza con ACE2 como en el SARS-CoV (Li et al., 2003) y S2 se funde con la membrana celular.

El estado conformacional predominante del trímero de la proteína $\mathrm{S}$ se subdivide en tres dominios (Receptor Binding Domains -RBD-), los que rotan para ubicarse en la posición adecuada para el enlace con su receptor (Wrapp et al., 2020).

\section{Estructura de RdRp}

La enzima RdRp o nsp12 (non structural protein 12), es la estructura esencial para el funcionamiento de la maquinaria que regula los procesos de replicación y transcripción del SARS-CoV-2.

RdRp sintetiza una cadena de ARN con orientación negativa $\left(3^{\prime}-5^{\prime}\right)$ que funciona como molde para crear los ARN con orientación positiva (5'-3') que posteriormente se traducirán en las proteínas estructurales del virus (Liu et al., 2020). Adicionalmente, cataliza la síntesis del ARN viral al formar un complejo con las enzimas no estructurales nsp7 y nsp8 (Gao et al., 2020).

Estas enzimas son generadas en las primeras etapas del ciclo viral, al ser traducido el ARN viral dentro del ribosoma cuando es liberado al citoplasma.

Las regiones del genoma del SARS-CoV-2 ORF1 y ORFlab generan varias poliproteínas (nsp7, nsp8 y nsp12 entre otras) claves en los procesos de replicación y transcripción del virus (Holshue et al., 2020).

En la cadena $\mathrm{N}$-terminal de la enzima, posee un dominio $\beta$-hairpina, el que es el blanco de antivirales como remdesivir y favipiravir.

$\mathrm{Su}$ estructura, elucidada por microscopía crio-electrónica consta de tres dominios fundamentales; el right-hand o dominio de polimerasa (residuos S367 a F920), el dominio N-terminal (residuos D60 a R249) que adopta una conformación denominada $\mathrm{Ni}$ dovirus RdRp-associated nucleotidyl transferase (NiRAN) (residuos A4 a T28 y Y69 a R249) y el dominio $\beta$-hairpina (residuos D29 a K50) (Gao et al., 2020).

Ambos dominios se conectan vía una interfaz entre los residuos A250 a R365 y con el dominio $\beta$-hairpina (residuos D29 a K50), quien se encuentra ubicado entre los dos dominios mayores. La orientación del complejo nsp8-nsp12 es opuesta a nsp7-nsp8, similar al del SARS-CoV, el que incluso posee una similitud en un 82\% del complejo nsp7-nsp8-nsp12 (Mirza \& Froeyen, 2020).

Los residuos N215 a D218 de nsp12 forman una hoja plegada $\beta$ que al hacer contacto con el residuo V96 a A100 estabilizan su conformación, lo que resulta en la formación de una estructura tipo "barril" compacto de las cuatro cadenas polipeptídicas.

El dominio $\beta$-hairpina se encuentra entre los dominios NiRAN y polimerasa, lo que es clave para la estabilización de la estructura de la enzima (Mirza \& Froeyen, 2020).

$\mathrm{Al}$ inhibirse a $\beta$-hairpina, se desestabiliza la estructura de nsp12 y por ende del complejo nsp7-nsp8nsp12, dejando de funcionar correctamente en los procesos de transcripción y replicación viral. Derivado a que tanto remdesivir como favipiravir se unen al dominio de $\beta$-hairpina, se tiene esperanza de su actividad terapéutica contra el COVID-19 (Chen et al., 2020).

Estudios sobre mutaciones del SARS-CoV-2 y su efecto sobre la estructura de RdRp indican que ha sufrido pequeños cambios, especialmente en su superficie, llamando la atención uno en su dominio de interfase, clave en las interacciones proteína-proteína. (Pachetti et al., 2020).

\section{Estructura de "3C-like protease" $\left(\mathrm{M}^{\mathrm{PRO}} \mathrm{o}\right.$ $3 C L^{\text {PRO) }}$}

$\mathrm{M}^{\mathrm{PRO}}$ o $3 \mathrm{CL}^{\mathrm{PRO}}$ es una enzima clave para la subsistencia y reproducción del SARS-CoV-2 al jugar un rol vital en sus procesos de replicación y transcripción.

La enzima de $33.8 \mathrm{kDa}$ (306 aminoácidos), es responsable de generar los polipéptidos funcionales del virus vía un extenso proceso proteolítico, que se inicia cuando es escindida por las poliproteinas la y lab (ppla y pplb).

$\mathrm{M}^{\mathrm{PRO}}$ al inicio del ciclo vital del virus dentro del citoplasma celular, genera 12 proteínas no estructurales (nsp4-nsp16), incluyendo a ARN-dependiente ARN polimerasa (RdRp, nsp12) y helicasa (nsp13), enzima que convierte al ARN viral de su sentido negativo (3'$\left.5^{\prime}\right)$ a positivo $\left(5^{\prime}-3^{\prime}\right)$ para facilitar su proceso de transcripción-replicación (Rut et al., 2020).

La escisión catalítica se realiza entre glutamina de posición P1 y serina, alanina o glicina en posición P1', actuando en 11 sitios conservados de la poliproteina viral (Jin et al., 2020).

Su sitio activo lo compone la pareja cisteína-histidina, en donde el azufre de la cisteína actúa como nucleófilo y el anillo imidazólico de histidina como donador de electrones. También juega un papel importante leu-gln, que puede intercambiar a gln por ser, ala o gli en el sitio activo funcional de la enzima (Zhang et al., 2020). 
La estructura cristalina de $\mathrm{M}^{\mathrm{PRO}}$ del SARS-CoV-2 es muy parecida a la del SARS-CoV (96\%), sin embargo, es $1,900 \%$ más sensible en su actividad derivado de los pequeños cambios estructurales a lo largo de toda la enzima y principalmente en interacciones de largo alcance. Estas se localizan alrededor de Cis-145 en el sitio catalítico y es el principal objetivo del diseño de inhibidores contra la enzima.

$\mathrm{M}^{\mathrm{PRO}}$ ha sido cristalizada de forma aislada y en complejo con inhibidores, estando disponibles sus estructuras en el sitio RCSB Protein Databank, su secuencia aminoacídica en NCBI (Chain A, SARSCoV-2 main protease) y con identificación P0DTD1 en la base de datos Protein Data Bank (Ullrich \& Nitsche, 2020).

Los dominios I y II (residuos 10-99 y 100-182), son seis hojas $\beta$ antiparalelas que contienen el sitio activo de la enzima y el objetivo de las estrategias terapéuticas para inhibirla (Bzówka et al., 2020; Estrada, 2020).

El dominio III (residuos 198-303) es un cluster globular de cinco hélices a que juegan un papel importante en regular la dimerización de $\mathrm{M}^{\mathrm{PRO}}$. Lo hace vía la interacción dipolar entre glu-290 y arg-4 de los protómeros de los dominios I y II respectivamente. El dímero que forma $\mathrm{M}^{\mathrm{PRO}}$ se mantiene unido por una interfase de $1394 \AA$, ubicada principalmente en el dominio II de la enzima (Zhang et al., 2020).

La importancia funcional de esta enzima dentro del ciclo de vida del virus y la ausencia de estructuras homólogas en humanos, hacen a $\mathrm{M}^{\mathrm{PRO}}$ un objetivo terapéutico atractivo para el diseño de medicamentos antivirales contra SARS-CoV-2 (Liu et al., 2020).

\section{Estudios in silico en búsqueda de nuevas moléculas contra SARS-CoV-2}

El descubrimiento de nuevas moléculas y la búsqueda de nuevas propiedades farmacológicas de moléculas ya existentes, son los principales objetivos de la farmacología moderna.

La secuenciación del genoma humano y su publicación en 2003, abrió oportunidad a la bioinformática de convertirse en una herramienta esencial en el análisis de la expresión y función génica (Leelananda \& Linder, 2016).

Avances en quimioinformática han permitido la creación de nuevos métodos computacionales para predecir actividad farmacológica y bibliotecas de com- puestos diseñados in silico (Terstappen \& Reggiani, 2001).

La bioinformática también ha contribuido a interpretar el genoma del SARS-CoV-2 y esclarecer su origen, al asociarlo estrechamente con el SARS-CoV obtenido de especies de murciélago, coadyuvando a la comprensión de su biología y develando futuros posibles tratamientos orientados hacia objetivos moleculares como la enzima convertidora de angiogtensina 2 (ACE2) y la trans membrana proteasa serina 2 (TMPRSS2), utilizadas por el virus para ingresar a la célula humana (Choudhury \& Mukherjee, 2020).

El enfoque in silico ha sido ampliamente adoptado en el descubrimiento de nuevos fármacos y utilizado en combinación con métodos quimio- y bioinformáticos para el estudio de oportunidades terapéuticas de moléculas derivadas de productos naturales (Chamdel et al., 2020; Lagunin et al., 2014).

La industria farmacéutica participa en el desarrollo de nuevas herramientas computacionales, incluyendo estudios de toxicidad, permitiendo modelar datos farmacocinéticos y la disminución en la utilización de animales de laboratorio (Kapetanovic, 2008).

La pandemia de COVID-19, generó un acelerado avance en la búsqueda de entidades farmacológicas que solas o combinadas, puedan ser una opción de tratamiento farmacológico.

El primer enfoque busca el análisis de moléculas ya disponibles en el mercado utilizadas contra otras afecciones, que puedan tener actividad contra el SARS-CoV-2 (Shah et al., 2020) y el segundo generar vía métodos de química computacional, compuestos derivados de moléculas existentes para desarrollar posibles nuevos fármacos.

Métodos in silico basados en homología, lograron elucidar el proteoma completo del virus, lo que permitió conocer su estructura y focalizar objetivos terapéuticos (Srinivasan et al., 2020).

Estudios bioinformáticos y filogenéticos han permitido comparar 15 genomas completos de SARS-CoV y SARS-CoV-2, encontrándose una similitud del 79.7\% entre ambos genomas y de forma más conservada las proteínas $\mathrm{E}$ y $\mathrm{N}$ en un 96 y $89.6 \%$ respectivamente, lo que permite orientar objetivos farmacológicos basados en evidencia de su efectividad frente al SARS-CoV (Zhou et al., 2020).

Los métodos in silico permiten un análisis amplio de moléculas en tiempo reducido, lográndose, por ejemplo, analizar 4,384 moléculas frente a $\mathrm{M}^{\mathrm{PRO}}$ del SARS-CoV-2, utilizando dinámica molecular y cal- 
culando su energía libre de unión a la proteína, identificándose moléculas como ergotamina y daunorubicina, para experimentación in vitro y ensayos clínicos (Jiménez-Alberto et al., 2020).

Ya en 2013 se reportan estudios in silico buscando inhibidores de TMPRSS2, proponiéndose varios derivados de amidino-bencilamida y de 4-sulfonil-3-aminofenilalanilamida con constantes de inhibición (Ki) de hasta $8 \mathrm{nM}$ (Meyer et al., 2013).

Recientemente Rahman y colaboradores (2020), estudiaron in silico la inhibición de metabolitos secundarios de plantas medicinales contra la enzima, resaltando compuestos como el lignano schisphenin A y el diterpeno de origen marino excavatolide M, mostrando valores de Docking Score de hasta -14.69 .

Sobre $\mathrm{M}^{\mathrm{PRO}}$, fueron analizadas moléculas aprobadas por FDA de Estados Unidos, donde ribavirina, telbivudina y las vitaminas B12 y nicotinamida fueron estudiadas por haber sido utilizadas contra SARS-CoV. El análisis se basó en la comparación bioinformática del receptor de la $\mathrm{M}^{\mathrm{PRO}}$ de SARS-CoV y SARS-Cov-2 fundamentada en la homología de los receptores, concluyendo en la posibilidad del uso de estos fármacos contra el virus (Kandeel \& Al-Nazawi, 2020).

Otro enfoque muy utilizado en técnicas in silico, es obtener moléculas de bancos de datos y generar posibles candidatos, los cuales pueden también ser optimizados para generar nuevas moléculas con menor toxicidad y mayor eficacia terapéutica.

Muchas de estas moléculas pueden tener origen en productos naturales como líquenes, de los cuales un cribado virtual, basado en su afinidad al receptor proteasa principal y cálculos farmacocinéticos, identificó a calicina y ácido rizocárpico con potenciales propiedades farmacológicas contra SARS-CoV-2 (Joshi, Sharma et al., 2020).

Otras moléculas de origen natural como la glicirricina, triptantrina y berberina, han mostrado también cierta evidencia de actividad in silico contra el virus (Narkhede et al., 2020).

También en la línea de productos naturales, la amentoflavona, un flavonoide presente en Ginkgo biloba L. ha despertado interés clínico derivado de una comparación in silico con fármacos como el antiviral ritonavir con resultados prometedores (Mishra et al., 2020). La hesperidina, otro flavonoide, también ha mostrado mayor efectividad que el antiviral nelfinavir frente $\mathrm{M}^{\mathrm{PRO}}$ in silico (Adam et al., 2020).

Capsaicina y piperina han mostrado plegamiento de 3CLproteasa in silico (Gonzalez-Paz et al., 2020)
Sin duda los productos naturales son una línea importante de investigación en búsqueda de moléculas con actividad contra COVID-19 (Khaerunnisa et al., 2020).

Algunos componentes de la miel también han sido estudiados, el ácido 3-fenil-láctico mostró fuerte interacción contra $\mathrm{M}^{\mathrm{PRO}}$ y actividad inhibitoria contra el virus (Hashem, 2020).

Por otro lado, derivado de la alta biodiversidad, algunas regiones del mundo como China, India e incluso Guatemala, cuentan con productos naturales únicos de interés científico. Carnosol y rosmanol, presentes en especies nativas de la región mediterránea o bien Psidium guajava L. de origen americano, han demostrado en Indonesia que son moléculas de alto peso molecular con algunas propiedades farmacocinéticas de interés en posibles tratamientos contra COVID-19 (Erlina et al., 2020; Umesh et al., 2020).

El polifenol oolonghomobisflavan-A presente en el té de Camellia sinensis (L.) Kuntze posee actividad inhibitoria sobre $\mathrm{M}^{\mathrm{PRO}}$ del SARS-CoV-2, los resultados sobre su energía de enlace, lo posicionan para avanzar hacia estudios in vitro e in vivo (Bhardwaj, 2020; Ghosh et al., 2020).

En total, catorce flavonoides han sido identificados en múltiples estudios in silico e incluso in vitro, a la espera de proceder a ensayos clínicos (Peterson, 2020).

A pesar de las bondades del diseño in silico de fármacos, el éxito y reducción de tiempo en el desarrollo de nuevos fármacos debe estar acompañado de un profundo conocimiento del organismo a combatir, su ciclo de vida y objetivo terapéutico y una combinación de diferentes ensayos.

Un ejemplo es el estudio realizado con 10,000 moléculas obtenidas de bancos de datos e incluso fármacos aprobados por FDA para otras enfermedades, que arrojó resultados optimistas al mostrar actividad antiviral en el complejo $\mathrm{N} 3$ de $\mathrm{M}^{\mathrm{PRO}}$, clave en la replicación del virus. Los ensayos, además de in silico, fueron realizados por RT-PCR en líneas celulares y cristalografía por rayos $\mathrm{X}$ para determinar la estructura del sitio de unión entre la molécula y el receptor, además de su actividad (Jin et al., 2020).

Antivirales como nelfinavir, un antirretroviral aprobado para utilizarse contra el VIH, ha mostrado resultados positivos como inhibidor de $\mathrm{M}^{\mathrm{PRO}}$ del SARSCoV-2 (Kumar et al., 2020).

La flavina adenina dinucleótido (FAD), fármaco utilizado en deficiencia de coenzima $\mathrm{A}$, ha mostrado resultados prometedores en su comparación con el antiviral indinavir (Hall \& Ji, 2020). 
Los métodos in silico, también se utilizan para dilucidar mecanismos de acción clave para el posterior desarrollo de fármacos.

Un ejemplo es la interacción entre la proteína $\mathrm{S}$ del SARS-CoV-2 y los receptores nicotínicos, que, vía simulaciones in silico, explican su patogenicidad e infectividad (Oliveira et al., 2020).

$\mathrm{Si}$ bien la reproducibilidad en las investigaciones es fundamental, modificaciones en la metodología pueden arrojar distintos resultados, tal es el caso de 7,922 compuestos que fueron analizados frente a $\mathrm{M}^{\mathrm{PRO}}$ del virus, destacándose seis moléculas: lasinavir, brecanavir, telinavir, rotigaptido, pimelautida y 1,3-bis-(2-etoxicarbonilcromon-5-iloxi)-2-(lisiloxi)propano.

Las diferencias en la metodología utilizada, radican en la selección de ligandos, preparación de receptores para su análisis y criterios para determinar la fortaleza de la interacción (Durdagi, 2020).

Una revisión de literatura sobre cefuroxima, una cefalosporina de segunda generación, recopiló evidencia sobre su efectividad contra SARS-CoV-2 en tres receptores proteicos, $\mathrm{M}^{\mathrm{PRO}}$, ARN-dependiente ARN polimerasa y el complejo proteína S-ACE2, generando interés en investigar in vitro e incluso clínicamente sobre esta cefalosporina (Durojaiye et al., 2020).

Hasta ahora los métodos basados en el acoplamiento y medición de su energía de enlace, han presentado resultados interesantes, sin embargo, con la metodología Deep docking es posible evaluar miles de compuestos de forma ágil.

La plataforma permite modelar la relación cuantitativa estructura-actividad farmacológica (QSAR por sus siglas en inglés), la que unida a bibliotecas de bancos de datos como ZINC15, puede analizar de forma rápida más de un millón de moléculas (Gentile et al., 2020).

Deep docking junto con ZINC15 se utilizaron para evaluar 1.3 millones de moléculas contra $\mathrm{M}^{\mathrm{PRO}}$ de SARS-CoV-2, obteniéndose 1,000 moléculas como potenciales inhibidores (Ton et al., 2020).

Los inhibidores de VIH-proteasa, ritonavir y lopinavir han sido estudiados contra el COVID-19 con resultados desalentadores, incluso en estudios in silico, compuestos como la viomicina formó más puentes de hidrógeno y mostró mayor afinidad a la $\mathrm{M}^{\mathrm{PRO}}$ viral (Kumar et al., 2020; Mahanta et al., 2020).

Fármacos utilizados en primera línea contra enfermedades como la influenza pandémica A y B, también han sido ensayados in silico, algunos con resultados negativos, tal es el caso del oseltamivir, ineficaz clínicamente contra COVID-19 (Tan \& Jin, 2020).
Estudios in silico también buscan oportunidades para anticuerpos monoclonales humanizados como CR3022, que puede mejorar su interacción con $\mathrm{M}^{\mathrm{PRO}}$ con la sustitución de tres de sus aminoácidos en su estructura. Esto, derivado de la recién descubierta interacción entre sus dominios de enlace con el dominio receptor del SARS-CoV-2 y ACE2 (Corrêa et al., 2020).

Esta interacción ha sido elucidada gracias al estudio de la relación entre proteínas y genes que propone la existencia de 222 posibles, lo que abre un escenario para profundizar en mecanismos y fármacos potencialmente activos (Cava et al., 2020).

TMPRSS2 ha sido recientemente objeto de estudios para su inhibición, incluso in silico, con medicamentos como los mesilatos de nafamostat y camostat en búsqueda de posibles fármacos contra el COVID-19 (Hoffmann et al., 2020). Ambos poseen evidencia in vitro de inhibir a TMPRSS2 y están siendo estudiados en ensayos clínicos para ser utilizados contra el SARS-CoV-2.

En algunos países, nafamostat se utiliza como antiviral profiláctico y en cuadros de coagulopatía (Shrimp et al., 2020).

Algunos fármacos han ganado popularidad en los últimos meses como medicamentos que previenen o combaten al COVID-19, tal es el caso de ivermectina (mezcla 80/20 de sus formas bla como blb), presenta in silico afinidad a la enzima $3 \mathrm{Cl}^{\mathrm{PRO}}$ y su dominio HR2, lo que promete una opción sinérgica con otros fármacos contra el SARS-CoV-2 (Paz et al., 2020).

Sin embargo, recientemente estudios in vitro demostraron que la dosis requerida para atacar al virus es entre 50 a 100 veces mayor de la utilizada como antiparasitario humano y veterinario (Chaccour et al., 2020), por lo que no se recomienda su utilización.

La hidroxicloroquina (HQC), también ha causado polémica en su utilización debido a la falta de consenso sobre su efectividad derivada de resultados de estudios clínicos, sin embargo, desde la perspectiva in silico, HQC puede formar un enlace covalente con $\mathrm{M}^{\mathrm{PRO}}$ de SARS-Cov-2, lo que implica que, al modificarse su estructura para hacer este enlace más fuerte, puede disminuir su virulencia.

Mepacrina en el mismo estudio, mostró una mayor fortaleza de dicho enlace covalente (Jokhakar et al., 2020; Srivastava et al., 2020).

Paritaprevir, un fármaco utilizado para el tratamiento de hepatitis $\mathrm{C}$ se muestra prometedor (-10.73 $\mathrm{kcal} / \mathrm{mol}$ de energía de enlace con ACE2) en estudios in silico contra el COVID-19 (Kadioglu et al., 2020; Shah et al., 2020). 
Estudios in silico con 318 moléculas de origen fitoquímico, muestra a quercetina-3-vicianosida como la molécula de mayor afinidad al receptor $(-8.3 \mathrm{kcal} /$ mol para $\mathrm{M}^{\mathrm{PRO}}$ y $-11.0 \mathrm{kcal} / \mathrm{mol}$ para ACE2) en este estudio (Joshi, Joshi et al., 2020).

La estructura cristalina de $\mathrm{M}^{\mathrm{PRO}}$ de SARS-Cov-2 fue elucidada y con ello, fue posible observar la interacción con el antiviral nelfinavir, proponiéndose las modificaciones estructurales en la molécula para incrementar su potencia y efectividad, así como aumentar la comprensión sobre su mecanismo de acción (Huynh et al., 2020).

En un escenario sobre el diseño de nuevos fárma$\cos$, los antivirales glecaprevir y maraviroc, utilizados contra HIV, son candidatos a ser derivatizados en un potencial nuevo agente activo como inhibidores de $\mathrm{M}^{\text {PRO }}$ del SARS-Cov-2 (Shamsi et al., 2020).

Rubitecan y loprazolam han sido ensayados in silico como inhibidores de TMPRSS2, sin embargo, el compuesto ZINC000015988935 (-12.39 kcal/mol) en energía de inhibición para $\mathrm{M}^{\mathrm{PRO}}$ y $-4.64 \mathrm{kcal} / \mathrm{mol}$ en su enlace con TMPRSS2 por mecánica molecular y superficie de Poisson-Boltzmann ha mostrado ser un mejor candidato (Elmezayen et al., 2020).

Un nuevo candidato denominado GC376, con nombre comercial ANIV-19 ${ }^{\circledR}$, presenta mejor interacción con $\mathrm{M}^{\mathrm{PRO}}$ del SARS-CoV-2 (energía libre de enlace de $-51.59 \mathrm{kcal} / \mathrm{mol}$ ) y cuenta con datos de ensayos in vivo (Hung et al., 2020).

Yoshino y colaboradores (2020) del Instituto de Tecnología de Tokio, en sus estudios de simulaciones de dinámica molecular con $\mathrm{M}^{\mathrm{PRO}}$, identificaron a los aminoácidos His41, Gly143 y Glu166 como los residuos que interaccionan con los grupos funcionales comunes en péptidos, convirtiéndolos en un objetivo terapéutico contra el SARS-CoV-2.

Los fármacos antivirales más prometedores a la fecha por su avance en ensayos clínicos, son los inhibidores de la RdRp favipiravir (T-705) y remdesivir (GS-44152).

Ambos continúan siendo estudiados en ensayos clínicos de Fase III, mostrando favipiravir mayor efectividad y menos efectos secundarios, que lo convierten en el medicamento contra el COVID-19 más promisorio a la fecha (Naik et al., 2020; Wang et al., 2020).

El éxito terapéutico de un fármaco o combinación de fármacos que puedan ser utilizados en COVID-19, también depende de la anticipación a mutaciones que el virus pueda tener.

Por ello, es importante conocer el genoma y la secuencia completa del virus para determinar si el mis- mo ha mutado y cuáles son las estructuras virales que han sido modificadas, poniendo atención a proteína $\mathrm{S}$, $\mathrm{M}^{\mathrm{PRO}}$ y RdRp.

Métodos bioinformáticos actuales son capaces de comparar más de 4,000 genomas entre sí y relacionar sus variantes con mayor virulencia.

Recientemente se reporta que SARS-CoV-2 ha mutado desde marzo de 2020 de la cepa D614 a la G614 (Korber et al., 2020).

La mutación está en el cambio de ácido aspártico por glicina en la posición 614 del dominio A de la proteína $\mathrm{S}$ del virus, lo que facilita la escisión de dicha proteína en las dos subunidades S1 y S2.

Glicina no tiene la capacidad de establecer puentes de hidrógeno con la treonina de la posición 859 del dominio B. El ácido aspártico original lo hacía fuertemente. Al "perder" estos puentes de hidrógeno al incorporarse glicina, las dos subunidades de la proteína $\mathrm{S}$ se pueden "separar" más fácilmente haciendo más efectivo el proceso de ingreso del virus a la célula.

G614 tiene mayor capacidad para ingresar a la célula humana que D614 aunque su capacidad de transcripción, replicación y reproducción sigue siendo la misma.

Por ello en los países de América Latina, Estados Unidos de América y Europa Occidental en donde predomina la cepa G614, la tasa de mortalidad es mayor que en asiáticos con D614, ya que mientras más cantidad de virus hay dentro de la célula, los síntomas pueden aparecer más rápidamente y la eficacia de los tratamientos actuales, incluyendo terapia antiviral disminuye, requiriéndose una dosis mayor (Eaaswarkhanth et al., 2020).

La Tabla 1, muestra la estructura de las moléculas que mayor Energía de Enlace mostraron en estudios de inhibición in silico contra $\mathrm{M}^{\mathrm{PRO}}$.

Es evidente, entonces, la necesidad de profundizar en el estudio a nivel molecular de las estructuras del virus y sus mecanismos de ingreso a la célula humana, transcripción, replicación y traducción, para encontrar moléculas con efectividad terapéutica, en donde los estudios in silico, sin duda, juegan y jugarán un papel muy importante.

\section{Conclusiones}

Las técnicas de diseño in silico (in silico approach) de fármacos han sido utilizadas exitosamente en la búsqueda de nuevos medicamentos, generando una contribución importante en el desarrollo de nuevos 
Tabla 1

Estructura y valores de Energía de Enlace de Inhibidores in silico de $M^{P R O}$

\begin{tabular}{|c|c|}
\hline Molécula & $\Delta \mathrm{G}(\mathrm{kcal} / \mathrm{mol})$ \\
\hline Calicina & -8.4 \\
\hline Ácido Rizocárpico & -9.11 \\
\hline Glicirricina & -8.1 \\
\hline Triptantrina & -8.2 \\
\hline Berberina & -8.1 \\
\hline Amentoflavona & -9.13 \\
\hline Excavatolide M & -14.38 \\
\hline
\end{tabular}


Tabla 1 (Continuación)

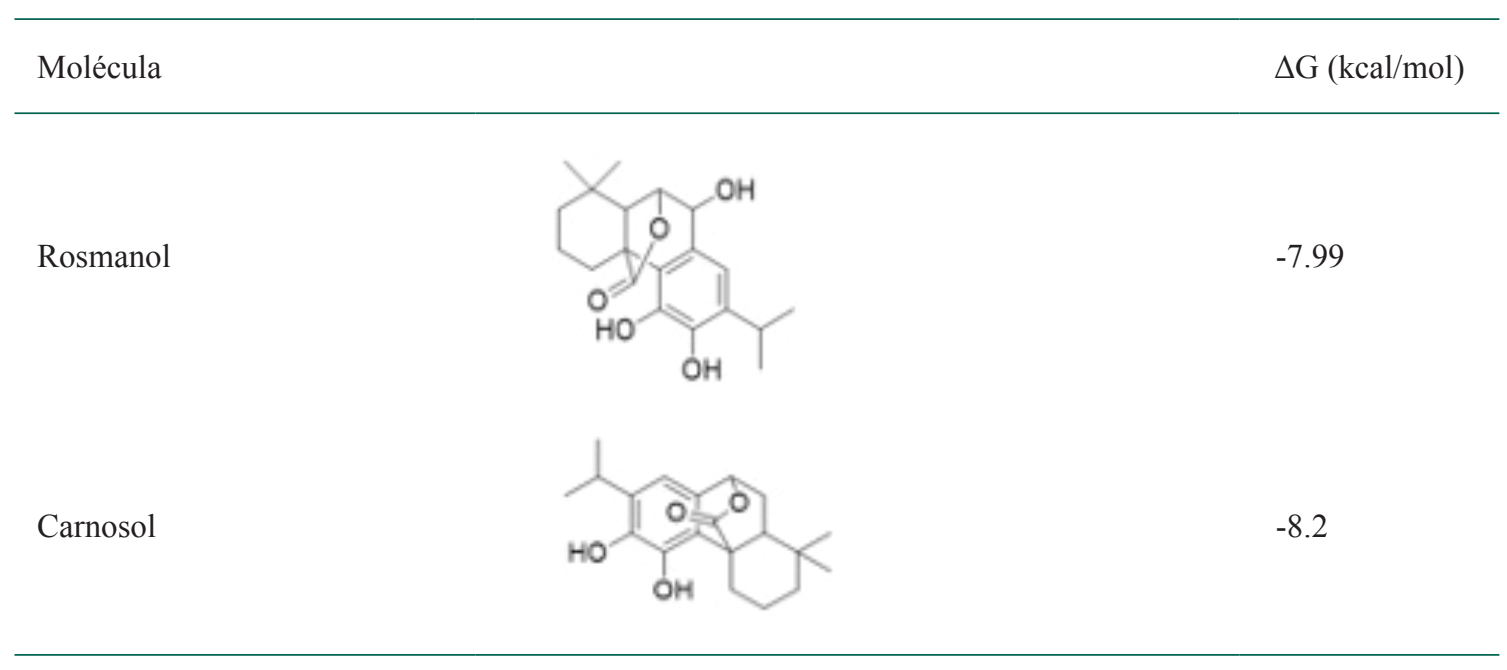

Nota. $\Delta \mathrm{G}$ representa la Energía Libre de Gibbs de la interacción entre la molécula y $\mathrm{M}^{\mathrm{PRO}}$ (Docking Score). Valor negativo más alto indica mayor fortaleza de enlace y por ende, mayor afinidad por el sitio activo de la enzima.

fármacos, al escalar moléculas a estudios in vitro y clínicos. Decenas de miles de compuestos; medicamentos actualmente en uso, derivados de productos naturales y moléculas modeladas, han sido estudiadas in silico en búsqueda de actividad contra el SARS-CoV-2, contribuyendo adicionalmente al entendimiento de sus mecanismos de acción. Se evidenció a lo largo de esta revisión la fortaleza de estas técnicas y su contribución en la búsqueda de moléculas capaces de inhibir a $\mathrm{M}^{\mathrm{PRO}}$, TRMPSS2 y proteína $\mathrm{S}$ como los principales objetivos terapéuticos en la búsqueda de una cura para COVID-19.

\section{Referencias}

Adam, S., Eyupoglu, V., Sarfraz, I., Rasuli, A., \& Ali, M (2020). Identification of potent Covid-19 main protease (Mpro) inhibitors from natural polyphenols: An in silico strategy unveils a hope against Corona. Preprints. https://doi. org/10.20944/preprint202003.0333.v1

Bhardwaj, R. (2020). A predictive model for the evolution of COVID-19. Transactions of the Indian National Academy of Engineering, 5, 133140. https://doi.org/10.1007/s41403-020-00130-w

Bianchi, M., Benvenuto, D., Giovanetti, M., Angeletti,
S., Ciccozzi, M., \& Pascarella, S. (2020). SARSCoV-2 envelope and membrane proteins: Differences from closely related proteins linked to cross-species transmission? BioMed Research International. Article 4389089. https://doi. org/10.1155/2020/4389089

Boopathi, S., Poma, A. B., \& Kolandaivel, P. (2020). Novel 2019 coronavirus structure, mechanism of action, antiviral drug promises and rule out against its treatment. Journal of Biomolecular Structure and Dynamics. https://doi.org/10.1080 /07391102.2020.1758788

Bosch, B. J., van der Zee, R., de Haan, C. A. M., \& Rottier, P. J. M. (2003). The Coronavirus spike protein is a class I virus fusion protein: Structural and functional characterization of the fusion core complex. Journal of Virology, 77(16), 8801-8811. https://doi.org/10.1128/JVI.77.16.8801-8811.2003

Bruno, A., Costantino, G., Sartori, L., \& Radi, M. (2019). The in silico drug discovery toolbox: Applications in lead discovery and optimization. Current Medicinal Chemistry, 26(21), 3838-3873. https:// doi.org/10.2174/0929867324666171107101035

Bzówka, M., Mitusińska, K., Raczyńska, A., Samol, A., Tuszyński, J., \& Góra, A. (2020). Structural and evolutionary analysis indicate that the 
SARS-CoV-2 $\mathrm{M}^{\mathrm{PRO}}$ is a challenging target for small-molecule inhibitor design. International Journal of Molecular Science, 21(9), Article 3099. https://doi.org/10.3390/ijms21093099

Cava, C., Bertoli, G., \& Castiglioni, I. (2020). In silico discovery of candidate drugs against Covid-19. Viruses, 12(4), Article 404. https://doi. org/10.3390/v12040404

Chaccour, C., Hamman, F., Ramón-García, S., \& Rabinovich, R. (2020). Ivermectin and COVID-19: Keeping rigor in times of urgency. The American Journal of Tropical Medicine and Hygiene, 102(6), 1156-1157. https://doi. org/10.4269/ajtmh.20-0271

Chamdel, V., Raj, S., Rathi, B. \& Kumar, D. (2020). In silico identification of potent Covid-19 main protease inhibitors from FDA approved antiviral compounds and active phytochemicals through molecular docking: A drug repurposing approach. Preprints. https://doi.org/10.20944/ preprints202003.0349.v1

Chen, C., Zhang, Y., Huang, J., Yin, P., Cheng, Z., Wu, J., ... Wang, X. (2020). Favipiravir versus arbidol for COVID-19: A randomized clinical trial. MedRxiv. https://doi. org/10.1101/2020.03.17.20037432

Choudhury, A., \& Mukherjee, S. (2020). In silico studies on the comparative characterization of the interactions of SARS-CoV-2 spike glycoprotein with ACE-2 receptor homologs and human TLRs. Journal of Medical Virology, 92(10). https://doi. org/10.1002/jmv.25987

Corrêa, C., Laaksonen, A., \& Barroso da Silva, F. L. (2020). On the interactions of the receptorbinding domain of SARS-CoV-1 and SARS$\mathrm{CoV}-2$ spike proteins with monoclonal antibodies and the receptor ACE2. Virus Research, 285, Article 198021. https://doi.org/10.1016/j. virusres.2020.198021

Durdagi, S. (2020). Virtual drug repurposing study against SARS-CoV-2 TMPRSS2 target. Turkish Journal of Biology, 44(3), 185-191. https://doi. org/10.3906/biy-2005-112

Durojaiye, A. B., Clarke, J.-R. D., Stamatiades, G. A., \& Wang, C. (2020). Repurposing cefuroxime for treatment of COVID-19: A scoping review of in silico studies. Journal of Biomolecular Structure
\& Dynamics. https://doi.org/10.1080/07391102.2 020.1777904

Eaaswarkhanth, M., Al Madhoun, A., \& Al-Mulla, F. (2020). Could the D614G substitution in the SARS-CoV-2 spike (S) protein be associated with higher COVID-19 mortality? International Journal of Infectious Diseases, 96, 459-460. https://doi.org/10.1016/j.ijid.2020.05.071

Elmezayen, A. D., Al-Obaidi, A., Şahin, A. T., \& Yelekçi, K. (2020). Drug repurposing for coronavirus (COVID-19): In silico screening of known drugs against coronavirus 3CL hydrolase and protease enzymes. Journal of Biomolecular Structure \& Dynamics. https://doi.org/10.1080/0 7391102.2020 .1758791

Estrada, E. (2020). Topological analysis of SARS CoV2 main protease. Chaos: An Interdisciplinary Journal of Nonlinear Science, 30(6), 061102. https://doi.org/10.1063/5.0013029

Erlina, L., Paramita, R. I., Kusuma, W. A., Fadilah, F., Tedjo, A., Pratomo, I. P., ... Yanur, A. (2020). Virtual screening on Indonesian herbal compounds as COVID-19 supportive therapy: Machine learning and pharmacophore modeling approaches. Research Square. https://doi. org/10.21203/rs.3.rs-29119/v1

Farag, A. B., Wang, P., Ahmed, M. S., \& Sadek, H. A. (2020). Identification of FDA approved drugs targeting COVID-19 virus by structure-based drug repositioning. ChemRxiv. https://doi. org/10.26434/chemrxiv.12003930.v1

Ganesan, A., Arimondo, P. B., Rots, M. G., Jeronimo, C., \& Berdasco, M. (2019). The timeline of epigenetic drug discovery: From reality to dreams. Clinical Epigenetics, 11(1), 174. https:// doi.org/10.1186/s13148-019-0776-0

Gao, Y., Yan, L., Huang, Y., Liu, F., Zhao, Y., Cao, L., ... Ge, J. (2020). Structure of RNA-dependent RNA polymerase from COVID-19 virus. Science, 368(6492), 779-782. https://doi.org/10.1126/ science.abb7498

Gentile, F., Agrawal, V., Hsing, M., Ton, A.-T., Ban, F., Norinder, U., ... Cherkasov, A. (2020). Deep docking: A deep learning platform for augmentation of structure based drug discovery. ACS Central Science, 6(6), 939-949. https://doi. org/10.1021/acscentsci.0c00229 
Ghosh, R., Chakraborty, A., Biswas, A., \& Chowdhuri, S. (2020). Evaluation of green tea polyphenols as novel corona virus (SARS CoV-2) main protease (Mpro) inhibitors - an in silico docking and molecular dynamics simulation study. Journal of Biomolecular Structure \& Dynamics, 1-13. https://doi.org/10.1080/07391102.2020. 1779818

Glowacka, I., Bertram, S., Müller, M. A., Allen, P., Soilleux, E., Pfefferle, S., ... Pöhlmann, S. (2011). Evidence that TMPRSS2 activates the severe acute respiratory syndrome coronavirus spike protein for membrane fusion and reduces viral control by the humoral immune response. Journal of Virology, 85(9), 4122-4134. https:// doi.org/10.1128/JVI.02232-10

Gonzalez-Paz, L. A., Lossada, C. A., Moncayo, L. S., Romero, F., Paz, J. L., Vera-Villalobos, J. ... Alvarado, Y. J. (2020). Theoretical molecular docking study of the structural disruption of the viral 3CL-protease of COVID19 induced by binding of capsaicin, piperine and curcumin Part 1: A comparative study with chloroquine and hydrochloroquine to antimalaric drugs. Research Square, preprint. https://doi.org/10.21203/rs-3rs-21206/v1

Hall, D. C., \& Ji, H.-F. (2020). A search for medications to treat COVID-19 via in silico molecular docking models of the SARS-CoV-2 spike glycoprotein and 3CL protease. Travel Medicine and Infectious Disease, 35, 101646. https://doi. org/10.1016/j.tmaid.2020.101646

Hashem, H. E. (2020). In Silico approach of some selected honey constituents as SARS-CoV-2 main protease (COVID-19) inhibitors. ChemRxiv. https://doi.org/10.26434/chemrxiv.12115359.v2

Hoffmann, M., Hofmann-Winkler, H., \& Pöhlmann, S. (2018). Priming time: How cellular proteases arm coronavirus spike proteins. En E. BöttcherFriebertshäuser, W. Garten \& H. D. Klenk (Eds.), Activation of Viruses by Host Proteases (pp. 7198). Cham: Springer. https://doi.org/10.1007/9783-319-75474-1_4

Hoffmann, M., Kleine-Weber, H., Krüger, N., Müller, M., Drosten, C., \& Pöhlmann, S. (2020). The novel coronavirus 2019 (2019$n \mathrm{CoV}$ ) uses the SARS-coronavirus receptor
ACE2 and the cellular protease TMPRSS2 for entry into target cells. BioRxiv. https://doi. org/10.1101/2020.01.31.929042

Holshue, M. L., DeBolt, C., Lindquist, S., Lofy, K. H., Wiesman, J., Bruce, H., ... Pillai, S. K. (2020). First case of 2019 novel coronavirus in the United States. New England Journal of Medicine, 382(10), 929-936. https://doi.org/10.1056/ NEJMoa2001191

Huang, Y., Yang, C., Xu, X.-F., Xu, W., \& Liu, S.-w. (2020). Structural and functional properties of SARS-CoV-2 spike protein: Potential antivirus drug development for COVID-19. Acta Pharmacologica Sinica, 41, 1141-1149. https:// doi.org/10.1038/s41401-020-0485-4

Hung, H.-C., Ke, Y.-Y., Huang, S. Y., Huang, P.-N., Kung, Y.-A., Chang, T.-Y., ... Hsu, J. T.-A. (2020). Discovery of $M$ protease inhibitors encoded by SARS-CoV-2. Antimicrobial Agents and Chemotherapy, 64, Article e200872. https:// doi.org/10.1128/AAC.00872-20

Huynh, T., Wang, H., \& Luan, B. (2020). In silico exploration of the molecular mechanism of clinically oriented drugs for possibly inhibiting SARS-CoV-2's main protease. Journal of Physical Chemistry Letters, 11(11), 4413-4420. https://doi.org/10.1021/acs.jpclett.0c00994

Jiménez-Alberto, A., Ribas-Aparicio, R. M., AparicioOzores, G., \& Castelán-Vega, J. A. (2020). Virtual screening of approved drugs as potential SARSCoV-2 main protease inhibitors. Computational Biology and Chemistry, 88, 107325. https://oi. org/10.1016/j.compbiolchem.2020.107325

Jin, Z., Du, X., Xu, Y., Deng, Y., Liu, M., Zhao, Y., ... Yang, H. (2020). Structure of $M^{\text {pro }}$ from SARSCoV-2 and discovery of its inhibitors. Nature, 582(7811), 289-293. https://doi.org/10.1038/ s41586-020-2223-y

Jokhakar, P. H., Kalaria, R., \& Patel, H. K. (2020). In silico docking studies of antimalarial drug hydroxychloroquine to SARS-CoV proteins: An emerging pandemic worldwide. ChemRxiv. https://doi.org/10.26434/chemrxiv.12488804.v1

Joshi, T., Joshi, T., Sharma, P., Mathpal, S., Pundir, H., Bhatt, V., \& Chandra, S. (2020). In silico screening of natural compounds against COVID-19 by targeting Mpro and ACE2 using 
molecular docking. European Review for Medical and Pharmacological Sciences, 24(8), 4529-4536. https://doi.org/10.26355/eurrev_202004_21036

Joshi, T., Sharma, P., Joshi, T., Pundir, H., Mathpal, S., \& Chandra, S. (2020). Structure-based screening of novel lichen compounds against SARS Coronavirus main protease (Mpro) as potentials inhibitors of COVID-19. Molecular Diversity. https://doi.org/10.1007/s11030-020-10118-x

Kadioglu, O., Saeed, M., Greten, H. J., \& Efferth, T. (2020). Identification of novel compounds against three targets of SARS CoV-2 coronavirus by combined virtual screening and supervised machine learning. [Preprint]. Bulletin of the World Health Organization. https://doi. org/10.2471/BLT.20.255943

Kaitin, K. I. (2010). Deconstructing the drug development process: The new face of innovation. Clinical Pharmacology \& Therapeutics, 87(3), 356-361. https://doi.org/10.1038/clpt.2009.293

Kandeel, M., \& Al-Nazawi, M. (2020). Virtual screening and repurposing of FDA approved drugs against COVID-19 main protease. Life Sciences, 251, 117627. https://doi.org/10.1016/j. lfs. 2020.117627

Kapetanovic, I. M. (2008). Computer-aided drug discovery and development (CADDD): In silico-chemico-biological approach. ChemicoBiological Interactions, 171(2), 165-176. https:// doi.org/10.1016/j.cbi.2006.12.006

Khaerunnisa, S., Kurniawa, H., Avaluddin, R., Suhartati, S., \& Soetjipto, S. (2020). Potential inhibitor of Covid-19 main protease ( $\left.M^{\text {pro }}\right)$ from several medicinal plant compounds by molecular docking study. Preprints. https://doi. org/10.20944/preprints202003.0226.v1

Kim, D., Lee, J.-Y., Yang, J.-S-, Kim, J.-W., Kim, V. N., $\&$ Chang, H. (2020). The architecture of SARSCoV-2 transcriptome. Cell, 181(4), 914-921.e10. https://doi.org/10.1016/j.cell.2020.04.011

Korber, B., Fischer, W., Gnanakaran, S., Yoon, H., Theiler, J., Abfalterer, W., ... Montefiori, D. (2020). Tracking changes in SARS-CoV-2 spike: Evidence that D614G increases infectivity of the COVID-19 virus. Cell, 182(4), 812-827.e19. https://doi.org/10.1016/j.cell.2020.06.043
Kumar, D., Chandel, V., Raj, S., \& Rathi, B. (2020). In silico identification of potent FDA approved drugs against Coronavirus COVID-19 main protease: A drug repurposing approach. Chemical Biology Letters, 7(3), 166-175. http://pubs.iscience.in/ journal/index.php/cbl/article/view/1033

Kumar, Y., Singh, H., \& Patel, C. N. (2020). In silico prediction of potential inhibitors for the main protease of SARS-CoV-2 using molecular docking and dynamics simulation based drugrepurposing. Journal of Infection and Public Health, 13(9), 1210-1223. https://doi.org/10.1016/j. jiph.2020.06.016

Lagunin, A. A., Goel, R. K., Gawande, D. Y., Pahwa, P., Gloriozova, T. A., Dmitriev, A. V., ... Poroikov, V. V. (2014). Chemo- and bioinformatics resources for in silico drug discovery from medicinal plants beyond their traditional use: A critical review. Natural Product Reports, 31(11), 1585-1611. https://doi.org/10.1039/C4NP00068D

Leelananda, S. P., \& Lindert, S. (2016). Computational methods in drug discovery. Beilstein Journal of Organic Chemistry, 12(1), 2694-2718. https://doi. org/10.3762/bjoc.12.267

Li, F., Li, W., Farzan, M., \& Harrison, S. C. (2005). Structure of SARS coronavirus spike receptorbinding domain complexed with receptor. Science, 309(5742), 1864-1868. https://doi. org/10.1126/science. 1116480

Li, G., \& De Clercq, E. (2020). Therapeutic options for the 2019 novel coronavirus (2019-nCoV). Nature Reviews Drug Discovery, 19(3), 149-150. https:// doi.org/10.1038/d41573-020-00016-0

Li, H., Zhou, Y., Zhang, M., Wang, H., Zhao, Q., \& Liu, J. (2020). Updated approaches against SARSCoV-2. Antimicrobial Agents and Chemotherapy, 64(6), Article e00483-20. https://doi.org/10.1128/ AAC.00483-20

Li, W., Moore, M. J., Vasilieva, N., Sui, J., Wong, S. K., Berne, M. A., ... Farzan, M. (2003). Angiotensinconverting enzyme 2 is a functional receptor for the SARS coronavirus. Nature, 426(6965), 450454. https://doi.org/10.1038/nature02145

Li, W., Zhang, C., Sui, J., Kuhn, J. H., Moore, M. J., Luo, S., ... Farzan, M. (2005). Receptor and viral determinants of SARS-coronavirus adaptation to 
human ACE2. The EMBO Journal, 24(8), 16341643. https://doi.org/10.1038/sj.emboj.7600640

Liu, C., Zhou, Q., Li, Y., Garner, L. V., Watkins, S. P., Carter, L. J., ... Albaiu, D. (2020). Research and development on therapeutic agents and vaccines for COVID-19 and related human Coronavirus diseases. ACS Central Science, 6(3), 315-331. https://doi.org/10.1021/acscentsci.0c00272

Lukassen, S., Chua, R. L., Trefzer, T., Kahn, N. C., Schneider, M. A., Muley, T., ... Eils, R. (2020). SARS-CoV-2 receptor ACE2 and TMPRSS2 are predominantly expressed in a transient secretory cell type in subsegmental bronchial branches. BioRxiv. https://doi. org/10.1101/2020.03.13.991455

Mahanta, S., Chowdhury, P., Gogoi, N., Goswami, N., Borah, D., Kumar, R., ... Gogoi, B. (2020). Potential anti-viral activity of approved repurposed drug against main protease of SARSCoV-2: An in silico based approach. Journal of Biomolecular Structure and Dynamics, 1-10. https://doi.org/10.1080/07391102.2020.1768902

Maranon, D. G., Anderson, J. R., Maranon, A. G., \& Wilusz, J. (2020). The interface between coronaviruses and host cell RNA biology: Novel potential insights for future therapeutic intervention. Wiley Interdisciplinary Reviews. $R N A, 11(5)$, Article e1614. https://doi.org/10.1002/ wrna.1614

Matsuyama, S., Nagata, N., Shirato, K., Kawase, M., Takeda, M., \& Taguchi, F. (2010). Efficient activation of the severe acute respiratory syndrome coronavirus spike protein by the transmembrane protease TMPRSS2. Journal of Virology, 84(24), 12658-12664. https://doi. org/10.1128/JVI.01542-10

Meyer, D., Sielaff, F., Hammami, M., BottcherFriebertshauser, E., Garten, W., \& Steinmetzer, T. (2013). Identification of the first synthetic inhibitors of the type II transmembrane serine protease TMPRSS2 suitable for inhibition of influenza virus activation. Biochemical Journal, 452(2), 331-343. https://doi.org/10.1042/ BJ20130101

Mirza, M. U., \& Froeyen, M. (2020). Structural elucidation of SARS-CoV-2 vital proteins: Computational methods reveal potential drug candidates against main protease, Nsp12 polymerase and Nsp13 helicase. Journal of Pharmaceutical Analysis, 10(4), 320-328. https:// doi.org/10.1016/j.jpha.2020.04.008

Mishra, A., Pathak, Y., Choudhir, G., Kumar, A., Mishra, S. K., \& Tripathi, V. (2020). Natural compounds as potential inhibitors of novel coronavirus (COVID-19) main protease: An in silico study. Research Square, preprint. https:// doi.org/10.21203/rs.3.rs-22839/v2

Monaghan, R. L., \& Barrett, J. F. (2006). Antibacterial drug discovery - Then, now and the genomics future. Biochemical Pharmacology, 71(7), 901909. https://doi.org/10.1016/j.bcp.2005.11.023

Naik, V. R., Munikumar, M., Ramakrishna, U., Srujana, M., Goudar, G., Naresh, P., ... Hemalatha, R. (2020). Remdesivir (GS-5734) as a therapeutic option of 2019-nCOV main protease - in silico approach. Journal of Biomolecular Structure \& Dynamics, 1-14. https://doi.org/10.1080/0739110 2.2020 .1781694

Narkhede, R. R., Pise, A. V., Cheke, R. S., \& Shinde, S. D. (2020). Recognition of natural products as potential inhibitors of COVID-19 main protease (Mpro): In-silico evidences. Natural Products and Bioprospecting, 10, 297-306. https://doi. org/10.1007/s13659-020-00253-1

Oliveira, A. S. F., Ibarra, A. A., Bermudez, I., Casalino, L., Gaieb, Z., Shoemark, D. K., ... Mulholland, A. J. (2020). Simulations support the interaction of the SARS-CoV-2 spike protein with nicotinic acetylcholine receptors and suggest subtype specificity. BioRxiv. https://doi. org/10.1101/2020.07.16.206680

Pachetti, M., Marini, B., Benedetti, F., Giudici, F., Mauro, E., Storici, P., ... Ippodrino, R. (2020). Emerging SARS-CoV-2 mutation hot spots include a novel RNA-dependent-RNA polymerase variant. Journal of Translational Medicine 18, Article 179. https://doi.org/10.1186/ s12967-020-02344-6

Paz, L. A. G., Lossada, C. A., Moncayo, L. S., Romero, F., Paz, J. L., Vera-Villalobos, ... Alvarado, Y. J. (2020). Molecular docking and molecular dynamic study of two viral proteins associated with SARS-CoV-2 with ivermectin. Preprints. https://doi.org/10.20944/preprints202004.0334.v1 
Peterson, L. E. (2020). COVID-19 and flavonoids: In silico molecular dynamics docking to the active catalytic site of SARS-CoV and SARS-CoV-2 main protease. Social Science Research Network. https://doi.org/10.2139/ssrn.3599426

Rahman, N., Basharat, Z., Yousuf, M., Castaldo G., Rastrelli, L., \& Khan, H. (2020). Virtual screening of natural products against type II transmembrane serine protease (TMPRSS2), the priming agent of Coronavirus 2 (SARS-CoV-2). Molecules, 25(10), 2271. https://doi.org/10.3390/ molecules 25102271

Rensi, S., Altman, R. B., Liu, T., Lo, Y.-C., McInnes, G., Derry, A., \& Keys, A. (2020). Homology modeling of TMPRSS2 yields candidate drugs that may inhibit entry of SARS-CoV-2 into human cells. ChemRxiv. https://doi.org/10.26434/ chemrxiv.12009582

Rut, W., Groborz, K., Zhang, L., Sun, X., Zmudzinski, M., Pawlik, B., ... Drag, M. (2020). Substrate specificity profiling of SARS-CoV-2 main protease enables design of activity-based probes for patient-sample imaging. BioRxiv. https://doi. org/10.1101/2020.03.07.981928

Sanders, J. M., Monogue, M. L., Jodlowski, T. Z., \& Cutrell, J. B. (2020). Pharmacologic treatments for coronavirus disease 2019 (COVID-19): A review. Journal of the American Medical Association, 323(18), 1824-1836. https://doi. org/10.1001/jama.2020.6019

Shah, B., Modi, P., \& Sagar, S. R. (2020). In silico studies on therapeutic agents for COVID-19: Drug repurposing approach. Life Sciences, 252, 117652. https://doi.org/10.1016/j.lfs.2020.117652

Shamsi, A., Mohammad, T., Anwar, S., Al Ajmi, M. F., Hussain, A., Rehman, M. T., ... Hassan, M. I. (2020). Glecaprevir and Maraviroc are highaffinity inhibitors of SARS-CoV-2 main protease: Possible implication in COVID-19 therapy. Bioscience Reports, 40(6), BSR20201256. https:// doi.org/10.1042/BSR20201256

Shrimp, J. H., Kales, S. C., Sanderson, P. E., Simeonov, A., Shen, M., \& Hall, M. D. (2020). An enzymatic TMPRSS2 assay for assessment of clinical candidates and discovery of inhibitors as potential treatment of COVID-19. BioRxiv. https://doi.org/10.1101/2020.06.23.167544
Shulla, A., Heald-Sargent, T., Subramanya, G., Zhao, J., Perlman, S., \& Gallagher, T. (2011). A transmembrane serine protease is linked to the severe acute respiratory syndrome Coronavirus receptor and activates virus entry. Journal of Virology, 85(2), 873-882. https://doi.org/10.1128/ JVI.02062-10

Sisay, M. (2020). Available evidence and ongoing clinical trials of remdesivir: Could it be a promising therapeutic option for COVID-19? Frontiers in Pharmacology, 11, 791. https://doi. org/10.3389/fphar.2020.00791

Srinivasan, S., Cui, H., Gao, Z., Liu, M., Lu, S., Mkandawire, W., ... Korkin, D. (2020). Structural genomics of SARS-CoV-2 indicates evolutionary conserved functional regions of viral proteins. Viruses, 12(4), 360. https://doi.org/10.3390/ v12040360

Srivastava, A. K., Kumar, A., Tiwari, G., Kumar, R., \& Misra, N. (2020). In silico investigations on the potential inhibitors for COVID-19 protease. ArXiv. arXiv:2003.10642v2

Tan, Q., \& Jin, Y. (2020). Oseltavimir is ineffective against COVID-19: In silico assessment, in vitro and retrospective study. MedRxiv. https://doi. org/10.1101/2020.05.15.20102392

Terstappen, G. C., \& Reggiani, A. (2001). In silico research in drug discovery. Trends in Pharmacological Sciences, 22(1), 23-26. https:// doi.org/10.1016/S0165-6147(00)01584-4

Ton, A.-T., Gentile, F., Hsing, M., Ban, F., \& Cherkasov, A. (2020). Rapid identification of potential inhibitors of SARS-CoV-2 main protease by deep docking of 1.3 billion compounds. Molecular Informatics, 39(8). https://doi.org/10.1002/ minf.202000028

Ullrich, S., \& Nitsche, C. (2020). The SARS-CoV-2 main protease as drug target. Bioorganic \& Medicinal Chemistry Letters, 30(17), 127377. https://doi.org/10.1016/j.bmcl.2020.127377

Umesh, Kundu, D., Selvaraj, C., Singh, S. K., \& Dubey, V. K. (2020). Identification of new antinCoV drug chemical compounds from Indian spices exploiting SARS-CoV-2 main protease as target. Journal of Biomolecular Structure \& Dynamics. https://doi.org/10.1080/07391102.2020. 1763202 
Wang, Y., Zhang, D., Guangua, G., Du, R., Zhao, J., Jin, Y., ...Wang, C. (2020). Remdesivir in adults with severe COVID-19: A randomised, double-blind, placebo-controlled, multicentre trial. Lancet, 395(10236), 1569-1578. https://doi.org/10.1016/ S0140-6736(20)31022-9

Weinmann, H., \& Metternich, R. (2005). Editorial: Drug discovery process for kinease inhibitors. ChemBioChem, 6(3), 455-459. https://doi. org/10.1002/cbic.200500034

Wrapp, D., Wang, N., Corbett, K. S., Goldsmith, J. A., Hsieh, C.-L., Abiona, O., ... McLellan, J. S. (2020). Cryo-EM structure of the 2019-nCoV spike in the prefusion conformation. Science, 367(6483), 1260-1263. https://doi.org/10.1126/ science.abb2507
Yoshino, R., Yasuo, N., \& Sekijima, M. (2020). Identification of key interactions between SARS-CoV-2 main protease and inhibitor drug candidates. Scientific Reports, 10, 12493. https:// doi.org/10.1038/s41598-020-69337-9

Zhang, L., Lin, D., Sun, X., Curth, U., Drosten, C., Sauerhering, L., ... Hilgenfeld, R. (2020). Crystal structure of SARS-CoV-2 main protease provides a basis for design of improved $\alpha$-ketoamide inhibitors. Science, 368(6489), 409-412. https:// doi.org/10.1126/science.abb3405

Zhou, Y., Hou, Y., Shen, J., Huang, Y., Martin, W., \& Cheng, F. (2020). Network-based drug repurposing for novel coronavirus $2019-\mathrm{nCoV} /$ SARS-CoV-2. Cell Discovery, 6(1), 1-18. https:// doi.org/10.1038/s41421-020-0153-3 